\title{
Finding the needle in the haystack: the diagnostic accuracy of the faecal immunochemical test for colorectal cancer in younger symptomatic patients
}

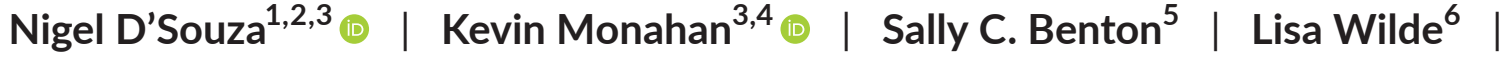 \\ Muti Abulafi ${ }^{1} \odot$ | the NICE FIT Steering Group* \\ ${ }^{1}$ Croydon University Hospital, Croydon, UK \\ ${ }^{2}$ University Hospital Southampton, Southampton, UK \\ ${ }^{3}$ Imperial College London, London, UK \\ ${ }^{4}$ St Mark's Hospital, Harrow, UK \\ ${ }^{5}$ Royal Surrey County Hospital, Guildford, UK \\ ${ }^{6}$ Bowel Cancer UK, London, UK
}

Correspondence

Muti Abulafi, Consultant Colorectal Surgeon, Croydon University Hospital, London Road, Croydon CR7 7YE, UK.

Email: muti.abulafi@nhs.net

Funding information

This study was funded by NHS England awarded to RM Partners, the West London Cancer Alliance hosted by the Royal Marsden NHS Foundation Trust. The study is supported by the National Institute for Health Research Clinical Research Network Portfolio. Croydon University Hospital acted as study sponsor. Alpha Labs Ltd, particularly Matthew Davis and Emma Boxall, supported the study by providing FIT collection devices without charge. All FIT kits and reagents were provided by Alpha Labs Ltd. The study sponsors and funders had no role in study design; in the collection, analysis and interpretation of data; in the writing of the report; and in the decision to submit the article for publication.

\begin{abstract}
Aim: Detection of early onset colorectal cancer is challenging, and remains a rare diagnosis amongst younger people with gastrointestinal symptoms. We investigated whether faecal immunochemical testing (FIT) could identify younger patients at higher risk of colorectal cancer or serious bowel disease including colorectal cancer, inflammatory bowel disease or advanced adenomas.

Methods: A subgroup analysis was performed of symptomatic patients under 50 years of age $(<50)$ from the NICE FIT study, a multicentre, prospective diagnostic accuracy study of FIT conducted between October 2017 and December 2019. The diagnostic accuracy of FIT for colorectal cancer and serious bowel disease was investigated in younger patients at different faecal haemoglobin ( $\mathrm{f}-\mathrm{Hb}$ ) cut-offs of 2, 10 and $150 \mu \mathrm{g}$ blood/g faeces $(\mu \mathrm{g} / \mathrm{g})$.

Results: Early onset colorectal cancer was diagnosed in 1.5\% (16/1103) of younger symptomatic patients. The sensitivity of FIT for younger patients aged $<50$ was $87.5 \%(95 \% \mathrm{Cl}$ $61.7 \%-98.4 \%), 81.3 \%(54.4 \%-96.0 \%)$ and $68.8 \%$ (41.3\%-89.0\%) at $\mathrm{f}-\mathrm{Hb}$ cut-offs of 2,10 and $150 \mu \mathrm{g} / \mathrm{g}$, respectively. The positive predictive value for colorectal cancer increased from $4.2 \%(2.3 \%-6.9 \%)$ to $11.5 \%(5.9 \%-19.6 \%)$ at cut-offs of 2 and $150 \mu \mathrm{g} / \mathrm{g}$, while the positive predictive value for serious bowel disease increased from $31.3 \%$ (26.3\%-36.5\%) to $65.6 \%(55.2 \%-75.0 \%)$ at the same cut-offs. The negative predictive value of FIT for colorectal cancer remained above $99.5 \%$ at all cut-offs.
\end{abstract}

*The members of the NICE FIT Steering Group are listed in the Acknowledgements.

TRIAL REGISTRATION: ISRCTN49676259.

This is an open access article under the terms of the Creative Commons Attribution License, which permits use, distribution and reproduction in any medium, provided the original work is properly cited.

(c) 2021 The Authors. Colorectal Disease published by John Wiley \& Sons Ltd on behalf of Association of Coloproctology of Great Britain and Ireland 
Conclusion: Detectable $\mathrm{f}-\mathrm{Hb}$ on $\mathrm{FIT}$ in symptomatic younger patients may indicate refer-

ral for investigation of colorectal cancer and serious bowel disease.

\section{KEYWORDS}

colonoscopy, colorectal neoplasms, diagnostic accuracy, faecal immunochemical test, FIT

\section{INTRODUCTION}

The identification of younger people at risk of colorectal cancer (CRC) represents a significant challenge in primary and secondary care. Whilst the absolute prevalence of CRC in younger people remains low, the disease incidence in this population is unfortunately increasing. Accurate risk assessment remains elusive given the poor predictive value of symptoms in younger people and as such novel approaches to risk stratification may be of significant clinical utility.

The incidence of early onset colorectal cancer (EOCRC), defined as a diagnosis of CRC under age 50 years, has significantly increased in recent years [1-6]. A contemporary global analysis [1] has reported an increase in the incidence of EOCRC in 19 out of 36 countries confirming similar data previously reported from countries including the UK [7], USA [3], Norway [4], Australia [5] and Canada [6]. Although the incidence of EOCRC is increasing, the absolute risk of CRC in this population remains low, with an incidence rate of 7.6 per 100000 in patients in England aged between 30 and 39, or 2.8 per 100000 in patients aged between 20 and 29 compared to 165.9 per 100000 in patients aged between 60 and 69 [8].

The poor predictive value of gastrointestinal symptoms provides an additional challenge in this population. Bowel symptoms in younger patients may be attributed by clinicians to common benign colorectal disease, such as rectal bleeding from haemorrhoids or abdominal pain from irritable bowel syndrome [9-11]. Unfortunately, younger patients who report non-specific symptoms but who have an underlying CRC may require multiple visits before referral for investigation, resulting in longer time to diagnosis [12-15]. A survey of EOCRC patients in the UK reported that $43 \%$ of patients required three or more visits to their primary care physician (PCP) before referral to secondary care for further investigation, and that $65 \%$ were initially given an incorrect diagnosis [11]. Patients themselves may not suspect their bowel symptoms may be due to cancer: in the same survey, $75 \%$ of patients did not suspect their symptoms were due to CRC, and 30\% delayed seeing their PCP for up to 3 months [11]. The interval from referral to diagnosis in younger patients is significantly longer in younger patients in national UK data, probably due to an absence of criteria indicating urgent referral for suspected cancer [12]. National cohort data also report that EOCRC more commonly presents as an emergency, at a more advanced stage of diagnosis $[12,14,16,17]$, although younger patients tend to have better survival than older patients $[18,19]$. It has been suggested that advanced-stage disease at presentation in younger patients may be due to delay in diagnosis, but there is also evidence that this may be due to a higher prevalence of biologically 'aggressive' tumours [16].

\section{What does this paper add to the literature?}

The faecal immunochemical test may indicate urgent investigation of younger patients with bowel symptoms; higher faecal haemoglobin concentrations (above $150 \mu \mathrm{g} / \mathrm{g}$ ) confer a $12 \%$ risk of colorectal cancer but also a $66 \%$ risk of serious bowel disease, including colorectal cancer, advanced adenomas and inflammatory bowel disease.

Thus the diagnosis of CRC in younger patients is difficult, and improvements in early diagnosis in this population may improve outcomes. We postulate that faecal immunochemical testing (FIT) may assist PCPs to identify which younger patients with higher risk symptoms may require colonoscopy or other diagnostics. FIT is a noninvasive, quantitative immunoassay which detects the globin moiety of haemoglobin in faeces and can reliably measure the faecal haemoglobin concentration ( $\mathrm{f}-\mathrm{Hb}$ ) to the nearest microgram of haemoglobin per gram of faeces $(\mu \mathrm{g} / \mathrm{g})$. To our knowledge, there are no published data specifically evaluating the diagnostic accuracy of FIT in younger patients referred urgently with suspected CRC symptoms.

\section{METHODS}

The primary end-point of this analysis was to establish the sensitivity of FIT for CRC in younger patients with symptoms of suspected CRC. The secondary end-points were to establish the diagnostic accuracy of FIT for CRC, advanced neoplasia (AN) (i.e., CRC and advanced adenoma $[A A])$ and serious bowel disease (SBD) (CRC, AA and inflammatory bowel disease [IBD]) in younger patients.

The NICE FIT study was a multicentre, ethically approved, diagnostic accuracy study of FIT in English patients referred urgently from primary care with symptoms of suspected CRC that were investigated with colonoscopy [20]. The study was designed with patient and public involvement, according to the standards of reporting of diagnostic studies (STARD) guidelines [21] and was registered prospectively (ISRCTN 49676259) after ethical approval by the National Research Ethics Committee on 23 January 2017 (reference 16/LO/2174). Patients were recruited at 50 National Health Service hospitals across England and took part in the study with informed consent. Symptom criteria for an urgent referral described by the National Institute for Health and Care Excellence (NICE) NG12 and DG30 criteria include change in bowel habit, iron deficiency anaemia, abdominal or rectal mass, and rectal bleeding $[22,23]$. When patients were referred 
with multiple symptoms, a previously reported hierarchy was used to match each patient to one symptom [20]. We defined younger patients as those referred urgently with symptoms under the age of 50 years $('<50$ '), with a comparator population (' $\geq 50$ ') of patients referred aged 50 years or older in line with published epidemiological data $[2,3,5]$.

The $\mathrm{f}-\mathrm{Hb}$ results on FIT measured on an HM-Jack analyser (Hitachi Chemical Diagnostics Systems Co. Ltd, Tokyo, Japan, supplied by Alpha Labs Ltd, Eastleigh, Hants, UK) were compared to colonoscopy findings. Data on patient demographics (age, sex, ethnicity, deprivation), serum haemoglobin and ferritin measurements and pathology results including tumour stage were also captured. AAs were defined in the study protocol as adenomas greater than $10 \mathrm{~mm}$, adenomas of any size with high grade dysplasia, and serrated polyps in the right colon. Patients with multiple findings at colonoscopy were re-categorized with one diagnosis in a hierarchy: CRC ranked highest followed by $\mathrm{AAs}$ and then IBD, followed by non-malignant findings. The diagnostic accuracy of FIT was calculated for CRC, AN and SBD. A full description of the methodology for the index test, reference standard, hierarchy of findings at colonoscopy and sample size calculation has been published previously [20].

Categorical data were compared with $\chi^{2}$ tests; the KruskalWallis test was used for ordinal or quantitative non-normal variables. Sensitivity, specificity, positive predictive value (PPV) and negative predictive value were reported for each $\mathrm{f}-\mathrm{Hb}$ cut-off, with $95 \%$ confidence intervals. Receiver operating characteristic (ROC) curves were plotted for $\mathrm{f}-\mathrm{Hb}$. These were done using an initial threshold of 0.1 to calculate sensitivity and specificity, and then recalculated with increments of 0.1 to plot the ROC curve. In every statistical analysis, $P<0.05$ was considered significant. All analyses were performed using Stata ${ }^{\circledR} 15$ (StataCorp 2017, Stata Statistical Software, Release 15; StataCorp LLC, College Station, Texas, USA).

\section{Role of the funding sources}

This study was funded by NHS England awarded to RM Partners, the West London Cancer Alliance hosted by the Royal Marsden NHS Foundation Trust. The study is supported by the National Institute for Health Research Clinical Research Network Portfolio. Croydon University Hospital acted as study sponsor. The study sponsors and funders had no role in study design; in the collection, analysis and interpretation of data; in the writing of the report; and in the decision to submit the article for publication.

\section{RESULTS}

In total, 9822 patients who underwent FIT for gastrointestinal symptoms were included in the final analysis, of whom 1103 were $<50$ years. The study flow is reported in Figure 1 and has been reported previously [20].

\section{Population description}

Patient demographics are reported in Table 1. The median age of patients in the $<50$ group was 44 years, and the median age in the $\geq 50$ group was 67 years. Women constituted $59.8 \%$ of patients $<50$ and $54.3 \%$ of patients $\geq 50$. Patients $<50$ were more ethnically diverse. Although the median index of deprivation was 6 in both groups, younger patients had a lower mean index of deprivation (5.7 vs. 6.3, $P<0.001$ ) and were significantly more likely to have a lower index of deprivation.

\section{Diagnostic yield at colonoscopy}

The findings at colonoscopy are reported in Table 2. The most common finding in all age groups was a normal colonoscopy (51.3\% of patients $<50,28.8 \%$ of patients $\geq 50$ ). CRC was significantly less commonly diagnosed in younger patients $(1.5 \%$ of patients $<50$ vs. $3.6 \%$ of patients $\geq 50, P<0.001$ ), as were AAs $(2.6 \%$ of patients $<50,4.5 \%$ of patients $\geq 50, P=0.004)$ and lower risk adenomas ( $13.1 \%$ of patients $<50,25 \%$ of patients $>50, P<0.001)$. IBD was significantly more commonly found in younger patients $(7.9 \%$ of patients $<50,3.9 \%$ of patients $\geq 50$, $P<0.001)$ and as a result there was no overall difference in the incidence of significant bowel disease (SBD $=I B D+A A+C R C)$, which was present in $12.0 \%$ (132/1103 vs. $1045 / 8719)$ in both populations.

\section{Diagnostic accuracy of FIT for CRC}

The diagnostic accuracy of FIT for CRC is reported in Table 3. The characteristics of all patients $<50$ diagnosed with CRC in this study are reported in Appendix S1. The sensitivity of FIT for patients aged $<50$ was $87.5 \%$ (95\% Cl 61.7\%-98.4\%), 81.3\% (54.4\%-96.0\%) and $68.8 \%(41.3 \%-89.0 \%)$ at cut-offs of 2,10 and $150 \mu \mathrm{g} / \mathrm{g}$, respectively. The PPV for CRC increased from $4.2 \%(2.3 \%-6.9 \%)$ to $11.5 \%$ $(5.9 \%-19.6 \%)$ at cut-offs of 2 and $150 \mu \mathrm{g} / \mathrm{g}$. The sensitivity of FIT for patients aged $\geq 50$ was $97.4 \%$ (95.0\%-98.9\%), 91.4\% (87.7\%$94.2 \%)$ and $70.9 \%(65.6 \%-75.9 \%)$ at cut-offs of 2,10 and $150 \mu \mathrm{g} / \mathrm{g}$, respectively.

The number needed to scope (NNS) to detect one CRC ranged from 23.8 in patients with detectable $\mathrm{f}-\mathrm{Hb}$ (above the $2 \mu \mathrm{g} / \mathrm{g}$ cut-off) to one CRC per 8.8 colonoscopies with $\mathrm{f}-\mathrm{Hb}$ measured above the $150 \mu \mathrm{g} / \mathrm{g}$ cut-off in patients <50. At the same FIT thresholds, the NNS was 10.9 and 2.9 in patients $\geq 50$.

When $\mathrm{f}-\mathrm{Hb}$ was undetectable $(<2 \mu \mathrm{g} / \mathrm{g}$ ), CRC prevalence (PPV) was less than $0.4 \%$ in all age groups, and the NNS ranged from 250 to 1000 .

On ROC curve analysis (Figure 2), the area under the curve (AUC) was $0.86(0.76-0.97)$ in patients $<50$ and $0.94(0.93-0.95)$ for patients $\geq 50$. 


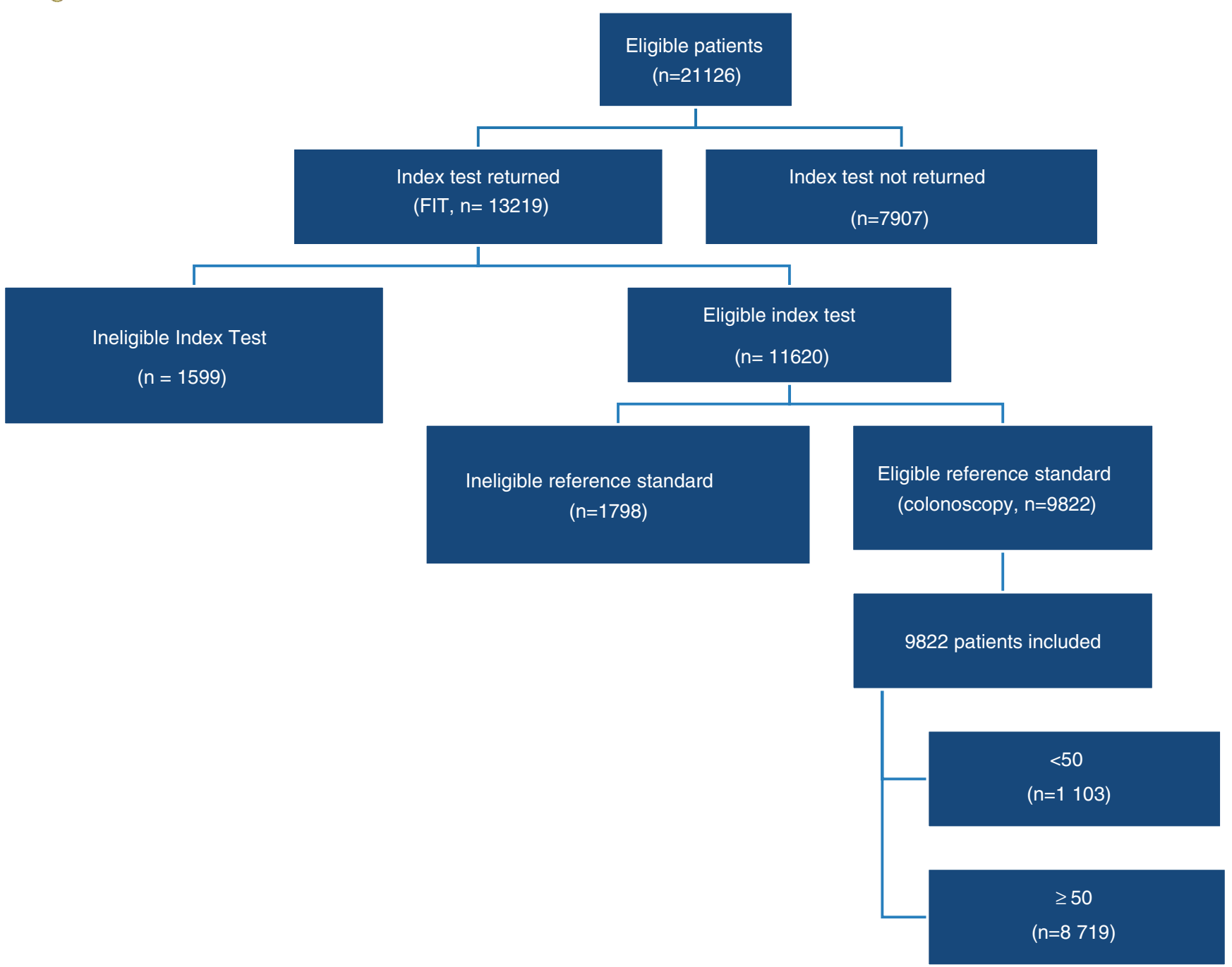

FIGURE 1 Study flow diagram. Ineligible index test: FIT performed after colonoscopy, FIT sample inadequate, FIT sample $>14$ days old. Ineligible reference standard: incomplete colonoscopy, underwent other investigations, cancelled colonoscopy, withdrew from study or missing data

\section{Diagnostic accuracy of FIT for AN}

The diagnostic accuracy of FIT for AN is reported in Table 4. The sensitivity of FIT for patients with AN aged $<50$ was $77.8 \%$ (62.9\%$88.8 \%), 64.4 \%(48.8 \%-78.1 \%)$ and $48.9 \%(33.7 \%-64.2 \%)$ at cut-offs of 2,10 and $150 \mu \mathrm{g} / \mathrm{g}$, respectively. The PPV for AN increased from $10.4 \%(7.4 \%-14.2 \%)$ to $22.9 \%(15.0 \%-32.6 \%)$ at cut-offs of 2 and $150 \mu \mathrm{g} / \mathrm{g}$. The sensitivity of FIT for patients aged $\geq 50$ was $79.6 \%$ (76.4\%-82.5\%), 65.4\% (61.7\%-68.9\%) and 43.1\% (39.4\%-46.9\%) at cut-offs of 2,10 and $150 \mu \mathrm{g} / \mathrm{g}$, respectively.

The NNS to detect one AN ranged from 9.6 in patients with detectable $\mathrm{f}-\mathrm{Hb}$ (above the $2 \mu \mathrm{g} / \mathrm{g}$ cut-off) to one AN per 4.4 colonoscopies with $\mathrm{f}-\mathrm{Hb}$ measured above the $150 \mu \mathrm{g} / \mathrm{g}$ cut-off in patients $<50$. At the same FIT thresholds, the NNS was 5.9 and 2.1 in patients $\geq 50$. On ROC curve analysis (Figure 3), the AUC of FIT for AN was 0.80 $(0.72-0.87)$ in patients $<50$ and $0.80(0.79-0.82)$ for patients $\geq 50$.

\section{Diagnostic accuracy of FIT for SBD}

The diagnostic accuracy of FIT for SBD is reported in Table 5. The PPV for SBD in patients $<50$ increased from $31.3 \%$ (26.3\%-36.5\%) to $65.6 \%(55.2 \%-75.0 \%)$ at cut-offs of 2 and $150 \mu \mathrm{g} / \mathrm{g}$. The PPV for SBD in patients $\geq 50$ increased from $24.2 \%(22.7 \%-25.7 \%)$ to $64.3 \%$ $(60.5 \%-68.0 \%)$ at the same cut-offs. The NNS to detect one case of SBD ranged from 3.2 in patients with detectable $\mathrm{f}-\mathrm{Hb}$ (above the $2 \mu \mathrm{g} / \mathrm{g}$ cut-off) to 1.5 with $\mathrm{f}-\mathrm{Hb}$ measured above the $150 \mu \mathrm{g} / \mathrm{g}$ cutoff in patients $<50$. At the same cut-offs the NNS was 4.1 and 1.6 in patients $\geq 50$.

In all age groups, when $\mathrm{f}-\mathrm{Hb}$ was undetectable $(<2 \mu \mathrm{g} / \mathrm{g}), \mathrm{SBD}$ prevalence (PPV) was less than $4.5 \%$ in all age groups, and the NNS ranged from 22.2 to 28.6. On ROC curve analysis (Figure 4), the AUC of FIT for SBD was $0.83(0.79-0.88)$ in patients $<50$ and $0.80(0.78-$ 0.81 ) for patients $\geq 50$. 
TABLE 1 Patient characteristics

\begin{tabular}{|c|c|c|c|c|c|c|}
\hline & & \multicolumn{2}{|c|}{$<50$ years } & \multicolumn{2}{|c|}{$\geq 50$ years } & \multirow[b]{2}{*}{$P$} \\
\hline & & $n$ & $\%$ & $n$ & $\%$ & \\
\hline \multirow[t]{3}{*}{ Age (years) } & Mean & 42 & & 66.7 & & \\
\hline & Standard deviation (SD) & 6.5 & & 9.16 & & \\
\hline & Maximum & 49 & & 97 & & \\
\hline \multirow[t]{2}{*}{ Sex } & Women & 659 & 59.8 & 4735 & 54.3 & 0.001 \\
\hline & Men & 444 & 40.2 & 3984 & 45.7 & \\
\hline \multirow[t]{4}{*}{ Ethnicity } & White & 685 & 68.4 & 6768 & 79 & $<0.001$ \\
\hline & Chinese & 12 & 1.2 & 29 & 0.3 & $<0.001$ \\
\hline & Other $^{a}$ & 192 & 18.1 & 911 & 10.6 & $<0.001$ \\
\hline & Missing & 49 & 4.4 & 138 & 1.4 & $<0.001$ \\
\hline \multirow[t]{3}{*}{ Index of deprivation } & Mean & 5.7 & & 6.3 & & $<0.001$ \\
\hline & Standard deviation (SD) & 2.6 & & 2.6 & & \\
\hline & Median & 6 & & 6 & & \\
\hline \multicolumn{7}{|l|}{ Referral symptom } \\
\hline \multicolumn{2}{|l|}{ Mass } & 30 & 2.7 & 147 & 1.7 & 0.02 \\
\hline \multicolumn{2}{|c|}{ Iron deficiency anaemia } & 65 & 5.9 & 414 & 4.8 & 0.10 \\
\hline
\end{tabular}

${ }^{a}$ Other ethnicity: any other ethnic group, not specified.

\section{DISCUSSION}

This is the first study to specifically report on the diagnostic accuracy of FIT for CRC in younger patients with suspected bowel cancer symptoms. The negative predictive value of FIT for CRC in our data is very high due to the low CRC prevalence in younger patients of $1.5 \%$. The sensitivity of FIT for CRC was moderate at lower cut-offs of 2 and $10 \mu \mathrm{g} / \mathrm{g}$, but the prevalence (i.e., PPV) was $11.5 \%$ at the highest cut-off of $150 \mu \mathrm{g} / \mathrm{g}$. However, FIT was associated with a high prevalence of SBD of $46.1 \%$ at the $10 \mu \mathrm{g} / \mathrm{g}$ cut-off recommended by NICE DG30. Even at a lower cut-off of detectable $\mathrm{f}-\mathrm{Hb}(>2 \mu \mathrm{g} / \mathrm{g})$, the prevalence of SBD was $31.3 \%$, rising to $65.6 \%$ at a cut-off of $150 \mu \mathrm{g} / \mathrm{g}$. This suggests that the presence of blood in faeces on FIT may indicate urgent investigation of symptomatic younger patients, particularly when $\mathrm{f}-\mathrm{Hb}$ measures above $150 \mu \mathrm{g} / \mathrm{g}$. Although our data may not support the use of FIT as a test to rule out CRC in younger patients, it can help clinicians in primary care to identify patients who require urgent referral and investigation for SBD, potentially an important application given the poor predictive value of symptoms alone for the identification of high risk patients. FIT at the time of initial presentation of younger patients with non-specific symptoms may also prevent delayed diagnosis due to further repeated attendances in primary care.

The role of heritable factors in EOCRC patients has been moderately well characterized, and provides significant opportunities for cancer prevention. However, the majority of EOCRC cannot currently be accounted for by known genetic syndromes, as pathogenic predisposition gene variants have been identified in only $15 \%-20 \%$ of patients [24]. In addition, only a minority of people with high risk conditions such as Lynch syndrome have been identified. A national CRC screening programme which incorporates all younger patients may not be feasible given the rarity of the disease in this population. While the American Cancer Society guidelines have advocated lowering the national screening age for CRC to 45 [25], this is based on an assumed increased CRC risk due to birth cohort effects that is not yet seen in real-world data. Furthermore, according to modelling 


\begin{tabular}{llllr}
\hline Diagnosis & $<50$ & $\geq 50$ & $P$ & Total \\
\hline Normal & $566(51.3 \%)$ & $2513(28.8 \%)$ & $<0.001$ & 3079 \\
Lower risk adenoma & $144(13.1 \%)$ & $2177(25.0 \%)$ & $<0.001$ & 2321 \\
Diverticular disease & $80(7.3 \%)$ & $2214(25.4 \%)$ & $<0.001$ & 2294 \\
Perianal disease & $166(15.1 \%)$ & $557(6.4 \%)$ & $<0.001$ & 723 \\
Microscopic colitis & $9(0.8 \%)$ & $143(1.6 \%)$ & 0.04 & 152 \\
Miscellaneous & $6(0.5 \%)$ & $47(0.5 \%)$ & 0.98 & 53 \\
Angiodysplasia & 0 & $23(0.3 \%)$ & 0.09 & 23 \\
Inflammatory bowel & $87(7.9 \%)$ & $340(3.9 \%)$ & $<0.001$ & 427 \\
$\quad$ disease & $29(2.6 \%)$ & $392(4.5 \%)$ & 0.004 & 421 \\
Advanced adenoma & $16(1.5 \%)$ & $313(3.6 \%)$ & $<0.001$ & 329 \\
Colorectal cancer & $132(12.0 \%)$ & $1045(12.0 \%)$ & 0.99 & 1177 \\
SBD (IBD + AA + CRC) & 1103 & 8719 & & 9822 \\
\hline Total & & & & \\
\hline
\end{tabular}

TABLE 2 Findings at colonoscopy by age group

Abbreviations: AA, advanced adenoma; CRC, colorectal cancer; IBD, inflammatory bowel disease; SBD, serious bowel disease.

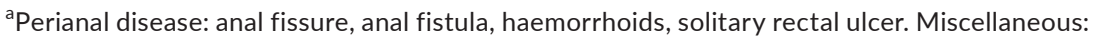
lipoma, melanosis coli, parasites.

TABLE 3 Diagnostic accuracy of FIT for CRC by age group

\begin{tabular}{|c|c|c|c|c|c|c|c|c|}
\hline Age group & $\begin{array}{l}\text { Cut-off } \\
(\mu \mathrm{g} / \mathrm{g})\end{array}$ & $\begin{array}{l}\text { FIT } \\
\text { positivity }\end{array}$ & $\begin{array}{l}\text { CRC per } \\
\text { cut-off }^{b}\end{array}$ & NNS & Sensitivity & Specificity & PPV & NPV \\
\hline \multirow[t]{7}{*}{$<50$ years } & None $^{a}$ & & $16 / 16$ & 68.9 & - & - & - & - \\
\hline & & & & & $(1.6-38.3)$ & $(26.9-32.4)$ & $(0.0-0.9)$ & (93.1-97.7) \\
\hline & $\geq 2$ & 38.1 & $14 / 16$ & 23.8 & 87.5 & 70.4 & 4.2 & 99.7 \\
\hline & $\geq 10$ & 19.2 & $13 / 16$ & 14.7 & 81.3 & 83.6 & 6.8 & 99.7 \\
\hline & & & & & $(54.4-96.0)$ & $(81.3-85.5)$ & (3.7-11.4) & $(99.0-99.9)$ \\
\hline & $\geq 150$ & 7.5 & $11 / 16$ & 8.7 & 68.8 & 92.2 & 11.5 & 99.5 \\
\hline & & & & & $(41.3-89.0)$ & (90.4-93.7) & $(5.9-19.6)$ & (98.8-99.8) \\
\hline \multirow{6}{*}{$\geq 50$ years } & $\geq 2$ & 38.1 & $305 / 313$ & 10.9 & 97.4 & 64.1 & 9.2 & 99.9 \\
\hline & & & & & $(95.0-98.9)$ & $(63.1-65.2)$ & $(8.2-10.2)$ & (99.7-99.9) \\
\hline & $\geq 10$ & 19.2 & $286 / 313$ & 5.8 & 91.4 & 83.5 & 17.1 & 99.6 \\
\hline & & & & & (87.7-94.2) & (82.7-84.3) & $(15.3-19.0)$ & $(99.4-99.7)$ \\
\hline & $\geq 150$ & 7.5 & $222 / 313$ & 2.9 & 70.9 & 94.9 & 34 & 98.9 \\
\hline & & & & & $(65.6-75.9)$ & (94.4-95.3) & (30.4-37.8) & $(98.6-99.1)$ \\
\hline
\end{tabular}

Note: $95 \%$ confidence interval within parentheses.

Abbreviations: CRC, colorectal cancer; FIT, faecal immunochemical test; NNS, number needed to scope; NPV, negative predictive value; PPV, positive predictive value, or prevalence.

${ }^{a}$ None is no FIT, i.e., all patients $<$ or $\geq 50$ years.

${ }^{b}$ Number of CRC detected at cut-off/number of CRC diagnosed in age group.

estimates, as the population screening age is lowered the relative risk of complications from colonoscopy increases alongside diminishing efficacy [26]. Pragmatically, many countries and populations do not have the endoscopy or financial resources to adopt such a screening strategy. The vast majority of EOCRC may most likely be diagnosed after presentation with symptoms. 


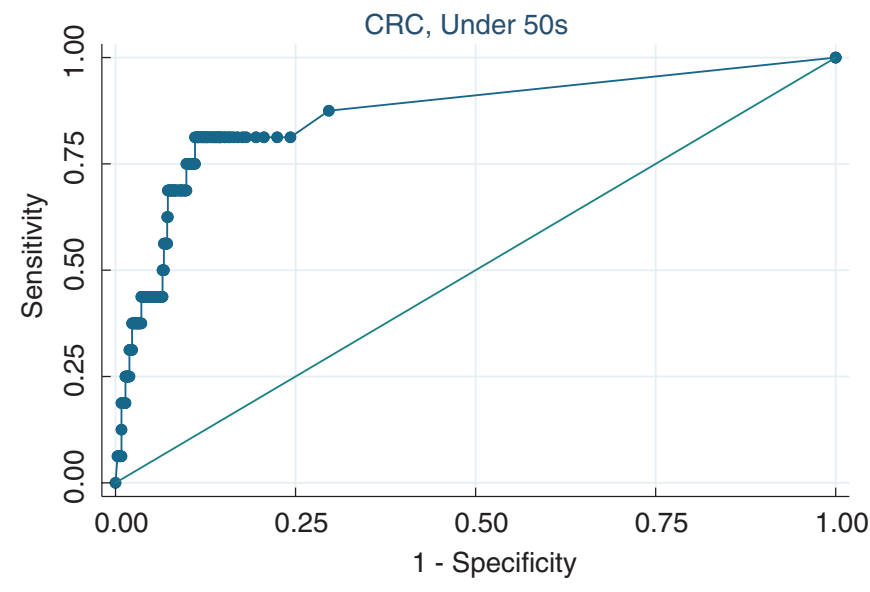

Area under ROC curve $=0.8649$

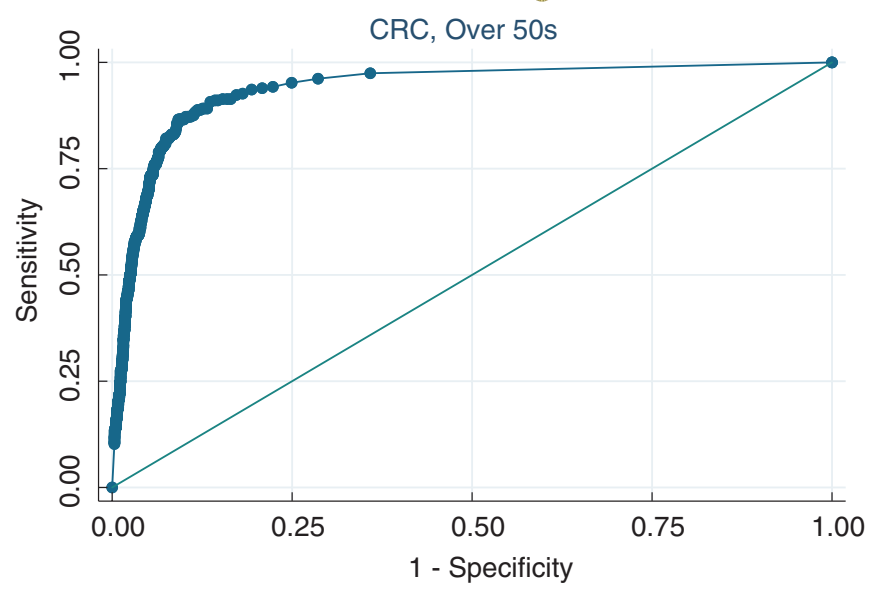

Area under ROC curve $=0.9383$

FIGURE 2 ROC curve analysis of FIT for CRC. Left: Patients aged $<50$ years. Right: Patients aged $\geq 50$ years

TAB LE 4 Diagnostic accuracy of FIT for AN by age group

\begin{tabular}{|c|c|c|c|c|c|c|c|c|}
\hline Age group & $\begin{array}{l}\text { Cut-off } \\
(\mu \mathrm{g} / \mathrm{g})\end{array}$ & $\begin{array}{l}\text { FIT } \\
\text { positivity }\end{array}$ & $\begin{array}{l}\text { AN per } \\
\text { cut-off }^{b}\end{array}$ & NNS & Sensitivity & Specificity & PPV & NPV \\
\hline \multirow[t]{7}{*}{$<50$ years } & None $^{a}$ & & $45 / 45$ & 24.5 & - & - & - & - \\
\hline & & & & & $(11.2-37.1)$ & $(25.7-31.3)$ & $(0.6-2.4)$ & $(85.8-92.6)$ \\
\hline & $\geq 2$ & 30.5 & $35 / 45$ & 9.6 & 77.8 & 71.6 & 10.4 & 98.7 \\
\hline & $\geq 10$ & 17.3 & $29 / 45$ & 6.6 & 64.4 & 84.7 & 15.2 & 98.2 \\
\hline & & & & & (48.8-78.1) & $(82.4-86.8)$ & $(10.4-21.1)$ & $(97.2-99.0)$ \\
\hline & $\geq 150$ & 8.7 & $22 / 45$ & 4.4 & 48.9 & 93.0 & 22.9 & 97.7 \\
\hline & & & & & $(33.7-64.2)$ & $(91.3-94.5)$ & $(15.0-32.6)$ & $(96.6-98.5)$ \\
\hline \multirow[t]{6}{*}{$\geq 50$ years } & None $^{a}$ & & $705 / 705$ & 12.3 & - & - & - & - \\
\hline & & & & & $(76.4-82.5)$ & $(64.5-66.6)$ & $(15.6-18.2)$ & $(96.9-97.7)$ \\
\hline & $\geq 10$ & 19.1 & $461 / 705$ & 3.6 & 65.4 & 84.9 & 27.6 & 96.5 \\
\hline & & & & & $(61.7-68.9)$ & (84.1-85.7) & $(25.5-29.8)$ & $(96.1-97.0)$ \\
\hline & $\geq 150$ & 7.5 & $304 / 705$ & 2.1 & 43.1 & 95.6 & 46.6 & 95.0 \\
\hline & & & & & $(39.4-46.9)$ & (95.2-96.1) & $(42.7-50.5)$ & (94.5-95.5) \\
\hline
\end{tabular}

Note: $95 \%$ confidence interval within parentheses.

Abbreviations: AN, advanced neoplasia; FIT, faecal immunochemical test; NNS, number needed to scope; NPV, negative predictive value; PPV, positive predictive value, or prevalence.

${ }^{a}$ None is no FIT, i.e., all patients $<$ or $\geq 50$ years.

${ }^{\mathrm{b}}$ Number of AN detected at cut-off/number of AN diagnosed in age group.

Colonoscopy remains the gold standard investigation of gastrointestinal symptoms and is increasingly employed in younger patients, predominantly to investigate symptoms [27]. Through identification and excision of adenomas and other precursors it is also an effective CRC preventative intervention [28]. In contrast, FIT is associated with a lower adenoma detection rate than colonoscopy, and thus is better designed to identify CRC rather than $A A$, as confirmed by our data where the false negative rate for AA was $54.6 \%$ at $10 \mu \mathrm{g} / \mathrm{g}$ [29]. The sensitivity of FIT for AN, with both CRC and AA, remained low in both younger and older patients in our data as a result of its low sensitivity to AA. As such there is only a moderate estimated benefit of colonoscopy as a 


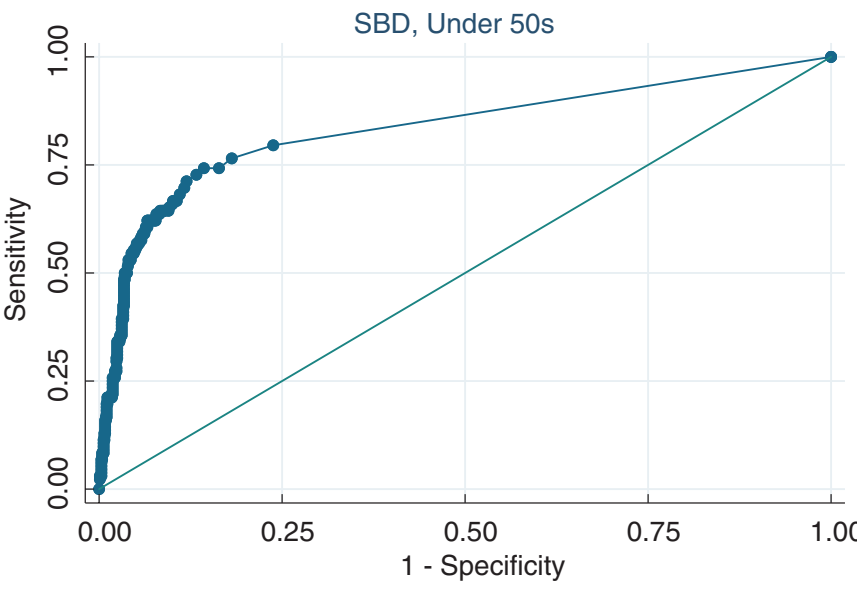

Area under ROC curve $=0.8344$

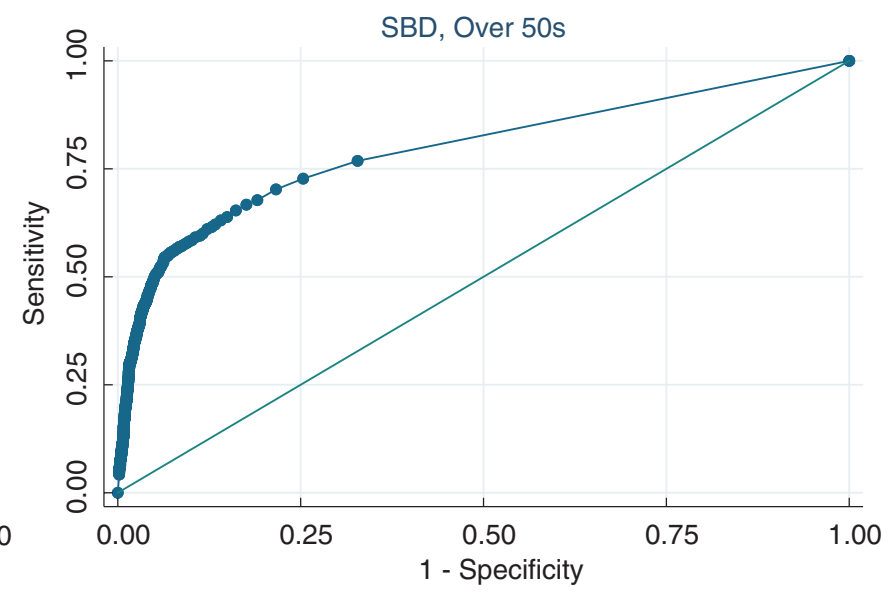

Area under ROC curve $=0.7955$

FIGURE 3 ROC curve analysis of FIT for AN. Left: Patients aged $<50$ years. Right: Patients aged $\geq 50$ years

TAB LE 5 Diagnostic accuracy of FIT for SBD by age group

\begin{tabular}{|c|c|c|c|c|c|c|c|c|}
\hline Age group & $\begin{array}{l}\text { Cut-off } \\
(\mu \mathrm{g} / \mathrm{g})\end{array}$ & $\begin{array}{l}\text { FIT } \\
\text { positivity }\end{array}$ & $\begin{array}{l}\text { SBD per } \\
\text { cut-off }^{\text {b }}\end{array}$ & NNS & Sensitivity & Specificity & PPV & NPV \\
\hline \multirow[t]{7}{*}{$<50$ years } & None $^{a}$ & & $132 / 132$ & 8.4 & - & - & - & - \\
\hline & & & & & (13.9-28.3) & $(21.1-26.6)$ & $(2.3-5.1)$ & $(63.5-73.7)$ \\
\hline & $\geq 2$ & 30.5 & $105 / 132$ & 3.2 & 79.5 & 76.2 & 31.3 & 96.5 \\
\hline & $\geq 10$ & 17.3 & $88 / 132$ & 2.2 & 66.7 & 89.4 & 46.1 & 95.2 \\
\hline & & & & & $(57.9-74.6)$ & (87.3-91.3) & (38.9-53.4) & $(93.6-96.5)$ \\
\hline & $\geq 150$ & 8.7 & $62 / 132$ & 1.5 & 47.7 & 96.6 & 65.6 & 93.1 \\
\hline & & & & & $(39.0-56.6)$ & $(95.3-97.6)$ & $(55.2-75.0)$ & (91.4-94.6) \\
\hline \multirow[t]{6}{*}{$\geq 50$ years } & $N_{0} e^{a}$ & & $1045 / 1045$ & 8.3 & - & - & - & - \\
\hline & & & & & $(74.2-79.4)$ & $(66.2-68.3)$ & $(22.7-25.7)$ & $(94.9-96.1)$ \\
\hline & $\geq 10$ & 19.2 & $649 / 1045$ & 2.6 & 62.1 & 86.7 & 38.8 & 94.4 \\
\hline & & & & & $(59.1-65.1)$ & $(85.9-87.4)$ & $(36.5-41.2)$ & $(93.8-94.9)$ \\
\hline & $\geq 150$ & 7.5 & $420 / 1045$ & 1.6 & 40.2 & 97 & 64.3 & 92.3 \\
\hline & & & & & $(37.2-43.2)$ & $(96.6-97.3)$ & $(60.5-68.0)$ & $(91.6-92.8)$ \\
\hline
\end{tabular}

Note: $95 \%$ confidence interval within parentheses.

Abbreviations: FIT, faecal immunochemical test; NNS, number needed to scope; NPV, negative predictive value; PPV, positive predictive value, or prevalence; SBD, serious bowel disease.

${ }^{a}$ None is no FIT, i.e., all patients $<$ or $\geq 50$ years.

${ }^{b}$ Number of SBD detected at cut-off/number of SBD diagnosed in age group.

preventative intervention in the younger symptomatic population compared to older individuals where AAs are more prevalent. In our study the absolute prevalence of CRC and AAs is low in symptomatic individuals under 50 years of age; as such FIT may be suitably employed in the identification of prevalent CRCs in this population. FIT may also offer more effective selection of patients who require colonoscopy, particularly where access to endoscopy may be limited during the COVID-19 pandemic.

Our results illustrate the difficulty in identifying CRC in younger symptomatic patients as only $1.5 \%$ of patients $<50$ were diagnosed with CRC. Symptoms are therefore poorly predictive for CRC in younger patients. Furthermore, it is assumed that only younger 


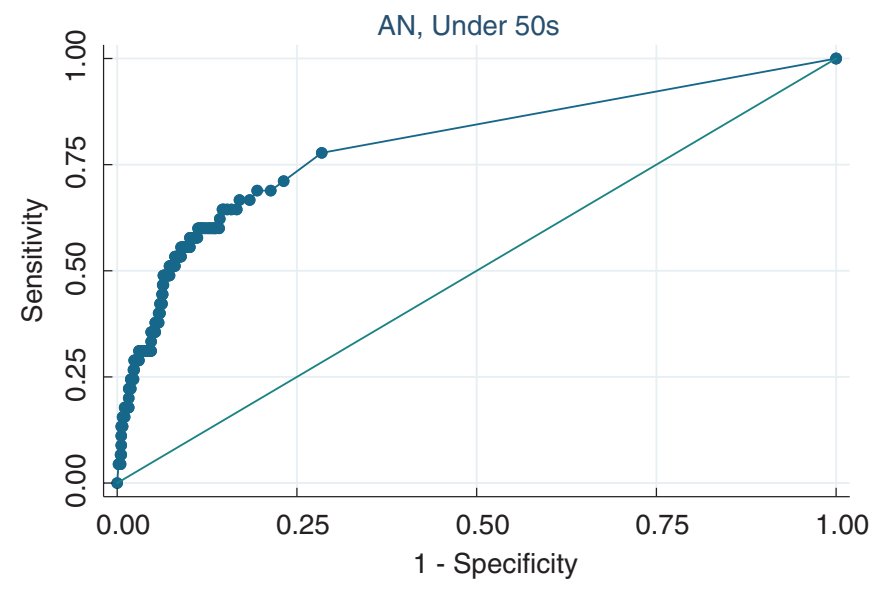

Area under ROC curve $=0.7968$

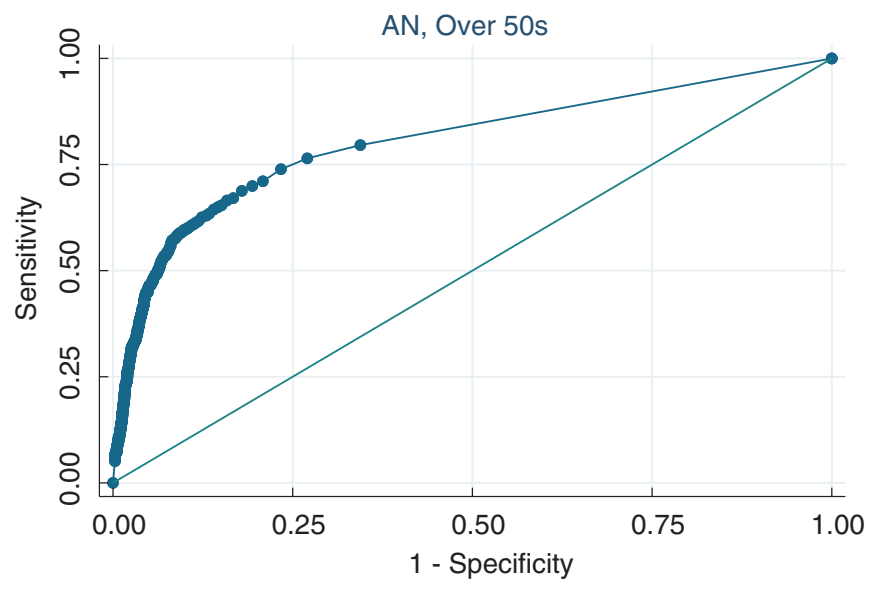

Area under ROC curve $=0.8041$

FIGURE 4 ROC curve analysis of FIT for SBD. Left: Patients aged $<50$ years. Right: Patients aged $\geq 50$ years

patients with the highest risk symptoms have been captured in this study, and that younger patients at even lower risk may have quite reasonably been managed in primary care and not referred for investigations. The primary end-point of the original NICE FIT study was powered to establish the sensitivity of FIT for CRC in patients referred urgently with suspected CRC symptoms and was not age stratified [20]. Consequently, this study is underpowered to detect the diagnostic accuracy of FIT in younger symptomatic patients. The prevalence of CRC may be lower in this group, and it is possible that triage for investigation with FIT may generate more false positive results. However, in the previous analysis of the NICE FIT study, triage of a group with lower risk (DG30) symptoms led to more true negative rather than false positive results [30]. There is no biological reason to suggest that the sensitivity of FIT will diminish in this population and fail to detect cancer. Further adequately powered research in a lower risk population may be required to determine the diagnostic accuracy of FIT for CRC or SBD, and also importantly the specificity and false positive rate, to avoid unnecessary anxiety to patients and unnecessary urgent referrals for investigation.

To establish accurate risk scores for younger symptomatic patients, prospective studies would need to be conducted in primary care to establish an accurate denominator, as only the highest risk patients will be referred on to secondary care. Due to the low prevalence of CRC in an unselected symptomatic younger population in primary care, this would require a very large study size. More objective measures alongside FIT, such as anaemia or thrombocytosis, may help to determine the risk of EOCRC [31, 32]. Further research into further non-invasive biomarkers for CRC such as breath [33] or urinary volatile organic compounds [34], or faecal/serum DNA samples, may help to identify patients at higher risk of CRC. Adding in faecal calprotectin into pathways for diagnosis of SBD may reduce false negative or false positive tests, although the detection of CRC and IBD was optimized by FIT without faecal calprotectin in a previous prospective study in primary care [35].
CRC diagnosis in younger symptomatic patients is difficult and FIT alone is unlikely to solve this problem. However, FIT could provide a diagnostic adjunct for PCPs to risk stratify referred patients in a similar way to how faecal calprotectin is currently being used. The higher prevalence of SBD in patients with elevated $\mathrm{f}-\mathrm{Hb}$ concentrations of $150 \mu \mathrm{g} / \mathrm{g}$ on FIT should indicate referral in younger patients and suggests that an urgent pathway should be set up for these patients, as this may avoid any delay to diagnosis in primary or secondary care [36].

\section{CONCLUSION}

FIT holds promise in the investigation of younger patients with bowel symptoms. Detectable $\mathrm{f}-\mathrm{Hb}$ should indicate referral for investigation of SBD, which will be detected in one in three patients. Higher $\mathrm{f}-\mathrm{Hb}$ concentrations above $150 \mu \mathrm{g} / \mathrm{g}$ confer a $12 \%$ risk of CRC and a $66 \%$ risk of SBD indicating urgent referral for investigation. Further work is required to assess the utility of FIT to risk stratify younger symptomatic patients who are likely to benefit from invasive investigations such as colonoscopy.

\section{AUTHOR CONTRIBUTORS}

NDS and MA were responsible for the conception and design of the study. NDS, KM, LW, SB and MA were responsible for data analysis and interpretation, and critical revision of the manuscript. The trial steering group provided oversight of recruitment activity, data analysis and interpretation. All authors were responsible for data acquisition. MA is the guarantor of the study, and affirms that the manuscript is an honest, accurate and transparent account of the study being reported; that no important aspects of the study have been omitted; and that any discrepancies from the study as originally planned (and, if relevant, registered) have been explained. 


\section{DATA AVAILABILITY STATEMENT}

Data will be shared with NHS England for further analysis but is not publicly available.

\section{ACKNOWLEDGEMENTS}

The study was supported by Croydon University Hospital and RM Partners in the design and conduct of the trial. Patients were recruited nationally through the NIHR Clinical Research Network, Principal Investigators and R\&D teams at each site. Alpha Labs Ltd, particularly Matthew Davis and Emma Boxall, supported the study by providing FIT collection devices without charge. All FIT kits and reagents were provided by Alpha Labs Ltd. The Southern Bowel Cancer Screening Hub Research Team provided analytical expertise and advice, in addition to analysing all samples. Hammersmith Medical Research provided expert database management and statistical analysis. Professor Callum G Fraser expertly revised drafts of the manuscript. Alpha Labs Ltd supported the study prior to funding with FIT kits and production of patient information.

The NICE FIT steering committee comprised: Theo Georgiou Delisle (Croydon University Hospital); Toni Mitchell (Hammersmith Medical Research); Kevin Monahan (West Middlesex University Hospital). The central study team included Anthonette DzakpasuAmevor and Christine Nangendo. Principal Investigators: Pasha Nisar (Ashford and St Peter's Hospitals NHS Foundation Trust); Yuksel Gercek (Bedford Hospital NHS Trust); Mark Austin (Brighton and Sussex University Hospitals NHS Trust); Nigel Hall (Cambridge University Hospitals NHS Foundation Trust); Robin Gupta (Chesterfield Royal Hospital NHS Foundation Trust); Rakesh Bhardway (Dartford and Gravesham NHS Trust); Arabis Oglesby (Dorset County Hospital NHS Foundation Trust); Usman Khan (East Cheshire NHS Trust); Nasser Khan (East and North Hertfordshire NHS Trust); Sudhakar Mangam (East Kent Hospitals University NHS Foundation Trust); Alexandra Cope (Frimley Health NHS Foundation Trust); Isobel Thomas (United Lincolnshire Hospitals NHS Trust); John Ramage (Hampshire Hospitals NHS Foundation Trust); Mark Pugh (Isle of Wight NHS Trust); Roshan Lal (James Paget University Hospitals NHS Foundation Trust); Tushar Agarwal, lan Jenkins (London North West University Healthcare); Mark Hill (Maidstone and Tunbridge Wells NHS Trust); Henk Wegstapel (Medway NHS Foundation Trust); Vimal Hariharan (North West Anglia NHS Foundation Trust); Salim Kurrimboccus (Pennine Acute Hospitals NHS Trust); Andrew Clarke (Poole Hospital NHS Foundation Trust); Tracey Dobson (Portsmouth Hospitals NHS Trust); Joseph Ellul (Princess Royal University Hospital, King's College Hospital NHS Foundation Trust); Hayley Webb (Queen Elizabeth Hospital Kings Lynn NHS Trust); Gemma Faulkner (Bolton NHS Foundation Trust); Sanjaya Wijeyekoon (Royal Bournemouth and Christchurch Hospitals NHS Foundation Trust); Neil Smart (Royal Devon and Exeter NHS Foundation Trust); John Stebbing (Royal Surrey NHS Foundation Trust); Jonathan Epstein (Salford Royal NHS Foundation Trust); Graham Branagan (Salisbury NHS Foundation Trust); Stephen Foley (Sherwood Forest Hospitals
NHS Foundation Trust); Andrew Bateman (University Hospital Southampton NHS Foundation Trust); Bruce Levy (Western Sussex Hospitals Foundation Trust); Andrew Day (Surrey and Sussex Healthcare NHS Trust); Fionnuala Lenehan (Walsall Healthcare NHS Trust); Nicholas Ward (West Suffolk NHS Foundation Trust); Lisa Wacklin (Weston Area Health NHS Trust); Naomi Mackenzie (Wrightington, Wigan and Leigh NHS Foundation Trust).

The NICE FIT Steering Group: Oliver Warren (Chelsea and Westminster Hospital NHS Foundation Trust); Saidyousuf Ahmadi, Theo Georgiou Delisle, Carlene Parchment, Arun Shanmuganandan (Croydon University Hospital); Nicholas West (Epsom and St Helier University Hospitals); Toni Mitchell, Stephen Sah and Nick Jackson (Hammersmith Medical Research); Alistair Myers (Hillingdon Hospitals NHS Foundation Trust); Paul Ziprin (Imperial College Healthcare NHS Trust); lan Bloom (Kingston Hospital NHS Foundation Trust); Michelle Chen, Stan Kaye (Royal Marsden Partners); Andy Ramwell (St George's University Hospitals NHS Foundation Trust); John T Jenkins (St Mark's Hospital); Kevin Monahan (West Middlesex University Hospital).

\section{CONFLICT OF INTEREST}

None.

\section{ETHICAL APPROVAL}

The study was approved by the National Research Ethics Service Committee, London South East (reference 16/LO/2174), and conducted with patient consent.

\section{ORCID}

Nigel D'Souza (D) https://orcid.org/0000-0002-9614-6113

Kevin Monahan (D) https://orcid.org/0000-0002-7918-4003

Muti Abulafi (D) https://orcid.org/0000-0001-7088-4292

\section{TWITTER}

Muti Abulafi @muti192

\section{REFERENCES}

1. Siegel RL, Torre LA, Soerjomataram I, Hayes RB, Bray F, Weber TK, et al. Global patterns and trends in colorectal cancer incidence in young adults. Gut. 2019;68(12):2179-85.

2. Araghi M, Soerjomataram I, Bardot A, Ferlay J, Cabasag CJ, Morrison DS, et al. Changes in colorectal cancer incidence in seven high-income countries: a population-based study. Lancet Gastroenterol Hepatol. 2019;4(7):511-8.

3. Siegel RL, Miller KD, Fedewa SA, Ahnen DJ, Meester RGS, Barzi A, et al. Colorectal cancer statistics, 2017. CA Cancer J Clin. 2017;67:177-93.

4. Larsen IK, Bray F. Trends in colorectal cancer incidence in Norway 1962-2006: an interpretation of the temporal patterns by anatomic subsite. Int J Cancer. 2010;126(3):721-32.

5. Young JP, Win AK, Rosty C, Flight I, Roder D, Young GP, et al. Rising incidence of early-onset colorectal cancer in Australia over two decades: report and review. J Gastroenterol Hepatol. 2015;30(1):6-13.

6. Patel $P, D e P$. Trends in colorectal cancer incidence and related lifestyle risk factors in 15-49-year-olds in Canada, 1969-2010. Cancer Epidemiol. 2016;42:90-100. 
7. Chambers AC, Dixon SW, White P, Williams AC, Thomas MG, Messenger DE. Demographic trends in the incidence of young-onset colorectal cancer: a population-based study. Br J Surg. 2020;107(5):595-605.

8. Exarchakou A, Donaldson LJ, Girardi F, Coleman MP. Colorectal cancer incidence among young adults in England: trends by anatomical sub-site and deprivation. PLoS One. 2019;14(12):e0225547.

9. Araujo SEA, Sousa Jr AHdSe, Campos FGCMd, Habr-Gama A, Dumarco RB, Caravatto PPdP, et al. Conventional approach $\times$ laparoscopic abdominoperineal resection for rectal cancer treatment after neoadjuvant chemoradiation: results of a prospective randomized trial. Rev Hosp Clin Fac Med Sao Paulo. 2003;58(3):133-40.

10. Myers EA, FeingoldDL, Forde KA, Arnell T, Jang JH, Whelan RL. Colorectal cancer in patients under 50 years of age: a retrospective analysis of two institutions' experience. World J Gastroenterol. 2013;19(34):5651-7.

11. Bowel Cancer UK. Never Too Young. 2020.

12. Arhi CS, Ziprin P, Bottle A, Burns EM, Aylin P, Darzi A. Colorectal cancer patients under the age of 50 experience delays in primary care leading to emergency diagnoses: a population-based study. Colorectal Dis. 2019;21(11):1270-8.

13. Scott RB, Rangel LE, Osler TM, Hyman NH. Rectal cancer in patients under the age of 50 years: the delayed diagnosis. Am J Surg. 2016;211(6):1014-8.

14. Chen FW, Sundaram V, Chew TA, Ladabaum U. Advanced-stage colorectal cancer in persons younger than 50 years not associated with longer duration of symptoms or time to diagnosis. Clin Gastroenterol Hepatol. 2017;15(5):728-37.e3.

15. Ben-Ishay O, Brauner E, Peled Z, Othman A, Person B, Kluger Y. Diagnosis of colon cancer differs in younger versus older patients despite similar complaints. Isr Med Assoc J. 2013;15(6):284-7.

16. Chou C-L, Chang S-C, Lin T-C, Chen W-S, Jiang J-K, Wang H-S, et al. Differences in clinicopathological characteristics of colorectal cancer between younger and elderly patients: an analysis of 322 patients from a single institution. Am J Surg. 2011;202(5):574-82.

17. Teng A, Lee DY, Cai J, Patel SS, Bilchik AJ, Goldfarb MR. Patterns and outcomes of colorectal cancer in adolescents and young adults. J Surg Res. 2016;205(1):19-27.

18. Burnett-Hartman AN, Powers JD, Chubak J, Corley DA, Ghai NR, McMullen CK, et al. Treatment patterns and survival differ between early-onset and late-onset colorectal cancer patients: the patient outcomes to advance learning network. Cancer Causes Control. 2019;30(7):747-55.

19. Abdelsattar ZM, Wong SL, Regenbogen SE, Jomaa DM, Hardiman $\mathrm{KM}$, Hendren S. Colorectal cancer outcomes and treatment patterns in patients too young for average-risk screening. Cancer. 2016;122(6):929-34.

20. D'Souza N, Georgiou Delisle T, Chen M, Benton S, Abulafi M. Faecal immunochemical test is superior to symptoms in predicting pathology in patients with suspected colorectal cancer symptoms referred on a $2 \mathrm{WW}$ pathway: a diagnostic accuracy study. Gut. 2021;70(6):1130-8. gutjnl-2020-321956.

21. Bossuyt PM, Reitsma JB, Bruns DE, Gatsonis CA, Glasziou PP, Irwig L, et al. STARD 2015: an updated list of essential items for reporting diagnostic accuracy studies. Radiology. 2015;277(3):826-32.

22. NICE. Suspected cancer: recognition and referral [NG12]. National Institute for Health and Care Excellence; 2015.

23. NICE. Quantitative faecal immunochemical tests to guide referral for colorectal cancer in primary care: diagnostics guidance [DG30]. National Institute for Health and Care Excellence. 2017.

24. Monahan KJ, Bradshaw N, Dolwani S, Desouza B, Dunlop MG, East JE, et al. Guidelines for the management of hereditary colorectal cancer from the British Society of Gastroenterology (BSG)/Association of Coloproctology of Great Britain and Ireland (ACPGBI)/United Kingdom Cancer Genetics Group (UKCGG). Gut. 2020;69(3):411-44.

25. Wolf AMD, Fontham ETH, Church TR, Flowers CR, Guerra CE, LaMonte SJ, et al. Colorectal cancer screening for average-risk adults: 2018 guideline update from the American Cancer Society. CA Cancer J Clin. 2018;68(4):250-81.

26. Peterse EFP, Meester RGS, Siegel RL, Chen JC, Dwyer A, Ahnen DJ, et al. The impact of the rising colorectal cancer incidence in young adults on the optimal age to start screening: microsimulation analysis I to inform the American Cancer Society colorectal cancer screening guideline. Cancer. 2018;124(14):2964-73.

27. Siegel RL, Fedewa SA, Anderson WF, Miller KD, Ma J, Rosenberg PS, et al. Colorectal cancer incidence patterns in the United States, 1974-2013. J Natl Cancer Inst. 2017;109(8):1974-2013. djw322.

28. Winawer SJ, Zauber AG, Ho MN, O'Brien MJ, Gottlieb LS, Sternberg SS, et al. Prevention of colorectal cancer by colonoscopic polypectomy. The National Polyp Study Workgroup. N Engl J Med. 1993;329(27):1977-81.

29. D'Souza NDT, Chen M, Benton S, Abulafi M, NICE FIT Steering Committee. Faecal immunochemical test is superior to symptoms in predicting pathology in patients with suspected colorectal cancer symptoms referred on a $2 \mathrm{WW}$ pathway; a diagnostic accuracy study. Gut. 2021;70:1130-8. in press.

30. D'Souza N, Georgiou Delisle T, Chen M, Benton S, Abulafi M. A faecal immunochemical test can guide referral of patients with high and low risk colorectal cancer symptoms; diagnostic accuracy results from 9822 patients in the NICE FIT Study. Br J Surg. 2021. Accepted for publication. https://doi.org/10.1093/bjs/znaa132.

31. Hamilton W, Lancashire R, Sharp D, Peters TJ, Cheng KK, Marshall $\mathrm{T}$. The importance of anaemia in diagnosing colorectal cancer: a case-control study using electronic primary care records. $\mathrm{Br} \mathrm{J}$ Cancer. 2008;98(2):323-7.

32. Bailey JA, Hanbali N, Premji K, Bunce J, Mashlab S, Simpson JA, et al. Thrombocytosis helps to stratify risk of colorectal cancer in patients referred on a 2-week-wait pathway. Int J Colorectal Dis. 2020;35:1347-50.

33. van Keulen KE, Jansen ME, Schrauwen RWM, Kolkman JJ, Siersema $\mathrm{PD}$. Volatile organic compounds in breath can serve as a non-invasive diagnostic biomarker for the detection of advanced adenomas and colorectal cancer. Aliment Pharmacol Ther. 2020;51(3):334-46.

34. Widlak MM, Neal M, Daulton E, Thomas CL, Tomkins C, Singh B, et al. Risk stratification of symptomatic patients suspected of colorectal cancer using faecal and urinary markers. Colorectal Dis. 2018;20(12):0335-O342.

35. Högberg C, Karling P, Rutegård J, Lilja M. Diagnosing colorectal cancer and inflammatory bowel disease in primary care: the usefulness of tests for faecal haemoglobin, faecal calprotectin, anaemia and iron deficiency. A prospective study. Scand J Gastroenterol. 2017;52(1):69-75.

36. Mitchell E, Macdonald S, Campbell NC, Weller D, Macleod U. Influences on pre-hospital delay in the diagnosis of colorectal cancer: a systematic review. Br J Cancer. 2008;98(1):60-70.

\section{SUPPORTING INFORMATION}

Additional supporting information may be found online in the Supporting Information section.

How to cite this article: D'Souza N, Monahan K, Benton SC, Wilde L, Abulafi M; the NICE FIT Steering Group. Finding the needle in the haystack: the diagnostic accuracy of the faecal immunochemical test for colorectal cancer in younger symptomatic patients. Colorectal Dis. 2021;00:1-11. https:// doi.org/10.1111/codi.15786 